\title{
IPAG - Interprofessionelle Zusammenarbeit auch bei eHealth
}

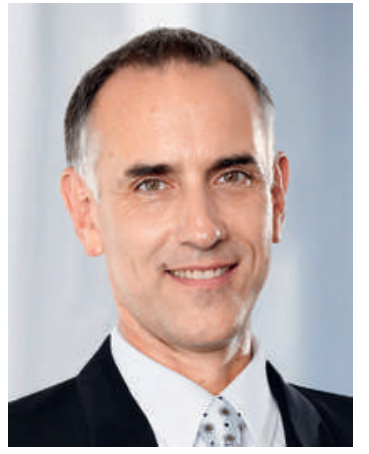

Im April dieses Jahres haben acht nationale Berufsverbände des Schweizer Gesundheitswesens - pharmaSuisse, der Schweizer Berufsverband der Pflegefachfrauen und Pflegefachmänner (SBK), ChiroSuisse, der ErgotherapeutInnen Verband Schweiz (EVS), der Schweizerische Verband diplomierter Ernährungsberater/innen $\mathrm{HF} /$ FH (SVDE), physioswiss sowie die FMH - die Interprofessionelle Arbeitsgruppe Elektronisches Patientendossier (IPAG EPD) gegründet. auf eine qualitativ hochwertige Versorgung zum Nutzen der Patienten abzielen.

Hierbei können die elektronischen Werkzeuge wertvolle Dienste leisten - vorausgesetzt, sie werden patientenorientiert und bedarfsgerecht ausgestaltet. Diese Bedürfnisse und Kenntnisse der Behandlungsprozesse will die IPAG EPD in die Entwicklung des elektronischen Patientendossiers (EPD) einbringen, um möglichst zielgerichtet vorzugehen.

Somit ist es die Hauptaufgabe der IPAG EPD, die Anliegen und Anforderungen der verschiedenen Berufsgruppen zu koordinieren, abzugleichen und in einer gemeinsam unterstützten Lösung zusammenzuführen. Dabei müssen die Inhalte im Mittelpunkt stehen; denn sie bilden die selbstverständliche Voraussetzung, um das anzustrebende Ziel «form follows function» zu erreichen.

\section{Verschiedene Berufsverbände des Schweizer Gesundheitswesens setzen sich für eine bedarfsgerechte Ausgestaltung des elektronischen Patientendossiers ein.}

Diese Kooperation ist - auch international betrachtet - ein bemerkenswerter Schritt, der deshalb z. B. auch bei der Konsultativtagung aller deutschsprachigen Ärzteorganisationen im Juni dieses Jahres auf grosses Interesse stiess. Die beteiligten Berufsverbände wollen hiermit ihren eigenen, professionell geprägten Beitrag zu den Entwicklungen im eHealth-Umfeld leisten. Die IPAG EPD möchte das elektronische Patientendossier als ein wichtiges Instrument zur Unterstützung patientenorientierter und berufsgruppenübergreifender Behandlungsprozesse aktiv fördern.

\section{eHealth soll die verschiedenen Berufsgruppen bei der Behandlung von Patienten unterstützen.}

Durch die Spezialisierung und die zunehmende Komplexität in den Behandlungsabläufen gewinnt die Kommunikation zwischen den einzelnen, an der Patientenbehandlung beteiligten Personen immer mehr an Bedeutung. Ein kranker Mensch erwartet von allen Beteiligten, dass sie ihre therapeutischen, pflegerischen, medizinischen und sonstigen Interventionen zu seinem Nutzen optimal aufeinander abstimmen. Interprofessionelle Zusammenarbeit sollte somit in erster Linie
Auch wenn anfänglich viel Vorbereitung und Engagement erforderlich sein werden, wird sich das EPD mit der interprofessionellen Arbeitsgruppe verschiedener Leistungserbringer effizienter und lösungsorientierter gestalten lassen. Grössere Reibungsverluste sowie zeitliche Verzögerungen, wie sie bei der separaten oder sukzessiven Befragung der verschiedenen relevanten Stakeholder vorkommen, lassen sich dank der IPAG EPD vermeiden.

An ihrer ersten Sitzung hat die Steuerungsgruppe der IPAG EPD den Auftrag erteilt, die inhaltlichen Arbeiten zu den beiden komplexen Themen «eAustrittsbericht» und «eMedikation» - gemäss der Prioritäten von eHealth Suisse - aufzunehmen. Unter der Federführung jeweils einer der grösseren Organisationen werden diese unterschiedlichen Themenbereiche gezielt angegangen. Eine kontinuierliche Abstimmung mit eHealth Suisse gehört ebenfalls zu den definierten Aufgaben der IPAG EPD.

Wir sind überzeugt, dass wir mit der IPAG EPD eine ausgezeichnete Idee realisiert haben, deren Erfolg nicht lange auf sich warten lassen wird. In der Kürze wird hier die Würze liegen - und allen Beteiligten ist klar: eHealth muss den Patienten und deren Behandelnden dienen!

Dr. med. Gert Printzen, Mitglied des Zentralvorstandes der FMH, Departementsverantwortlicher eHealth Medizinische Informatik und Dokumentation/Heilmittel 